Vázquez Toledo, S., Liesa Orús, M. \& Lozano Roy, A. (2017). Recreos Cooperativos e Inclusivos a través de la metodología de Aprendizaje-Servicio. Revista Electrónica Interuniversitaria de Formación del Profesorado, 20(1), 173-185.

\title{
Recreos Cooperativos e Inclusivos a través de la metodología de Aprendizaje-Servicio
}

Sandra Vázquez Toledo, Marta Liesa Orús y Azucena Lozano Roy

Facultad de Ciencias Humanas y de la Educación de Huesca - Universidad de Zaragoza

\section{Resumen}

Este artículo presenta una experiencia de aprendizaje-servicio llevada a cabo por la Facultad de Ciencias Humanas y de la Educación de Huesca. Dicha experiencia se ha materializado en un proyecto denominado Recreos cooperativos e inclusivos, cuya repercusión es trascendente puesto que se está implementando en siete centros públicos de la etapa de Educación Infantil y Primaria de la ciudad de Huesca. A partir de la misma hemos podido constatar como los estudiantes de Magisterio desarrollan competencias transversales del Grado, al mismo tiempo que brindan un servicio en el entorno próximo, mejorando las interacciones que se establecen en los recreos entre los niños de primaria y disminuyendo la exclusión social así como las dinámicas segregacionistas y marginadoras que se ponían de manifiesto en los recreos.

\section{Palabras clave}

Competencias transversales; aprendizaje-servicio; recreos; juegos cooperativos; inclusión

\section{Contacto:}

Sandra Vázquez Toledo, svaztol@unizar.es, C/ Valentín Carderera 4, 22003, Huesca. 


\title{
Cooperative and inclusive breaks through the service learning methodology
}

\begin{abstract}
This paper presents a service-learning experience conducted at the Faculty of Humanities and Education at the University of Zaragoza. This experience has resulted in a project called cooperative and inclusive breaks, whose impact is important because it is being carried out in almost all public schools in the Pre-School and Primary Education of the City of Huesca. In this project we have seen how students of the Teacher Degree have developed cross skills of the Degree, while they have provided a service in the immediate environment by 1 ) improving the interactions established during breaks between elementary school children and 2) reducing social exclusion and marginalizing dynamics that occur during breaks
\end{abstract}

\section{Keywords}

Cross competencies; service-learning; breaks; cooperative games; inclusion.

\section{Introducción}

La optimización de la calidad del aprendizaje académico de los estudiantes Universitarios y su formación integral como personas socialmente responsables, fin que en sí mismo se evidencia como uno de los primordiales de toda formación universitaria, es un tema con el que estamos comprometidos un grupo interdisciplinar de profesores de la Universidad de Zaragoza. Nuestra dedicación se centra en el desarrollo y divulgación de prácticas de "Aprendizaje-Servicio" en y desde nuestra institución. Para ello, hemos constituido un grupo de trabajo que a través de un proyecto de innovación -subvencionado por la Universidad de Zaragoza y respaldado por el Departamento de Educación, Universidad, Cultura y Deporte del Gobierno de Aragón-, trabaja en esta línea.

Estimamos que la metodología práctica de "Aprendizaje-Servicio", además de lograr mayor formación profesional y personal por parte de nuestros estudiantes, contribuye, aunque sea modestamente, a devolver a la sociedad la confianza que ha depositado en nosotros como organización promotora de conocimiento y de compromiso social.

Para ello proponemos procesos de enseñanza-aprendizaje en los que estén presentes experiencias que conlleven simultáneamente un aprendizaje y un servicio; un aprendizaje para el estudiante y un servicio a la comunidad. La experiencia práctica puede ser realizada con cualquier institución, asociación o entidad sin ánimo de lucro. En el caso que nos ocupa, en los CEIP públicos de la ciudad de Huesca, y con los Equipos de Orientación Educativa y Psicopedagógica que los atienden.

Para implementar esta metodología en el entorno próximo, hemos desarrollado proyectos como el de Recreos Cooperativos e Inclusivos. Este proyecto surgió como experiencia piloto el curso 2011-2012 en un colegio público de la ciudad ante la demanda del Equipo de Orientación Educativa y Psicopedagógica que atendía este centro. Actualmente dicho proyecto se ha solicitado desde todos los centros públicos de educación infantil y primaria de Huesca a excepción de uno.

En este proyecto optamos por metodologías cooperativas -juegos cooperativos en los recreos- ya que la cooperación lleva de forma implícita la inclusión, objetivo esencial del mismo. Coincidimos con Pujolàs (2012) en que la cooperación es un elemento clave a la hora 
de atender la diversidad del alumnado, porque se desarrolla la solidaridad y el respecto a las diferencias. $Y$ porque se potencian algunas competencias sociales -como diálogo, negociación, resolución de conflictos, resolver juntos problemas comunes, etc.- y competencias comunicativas -como, por ejemplo, expresar, argumentar e interpretar pensamientos, sentimientos y hechos; escuchar las ideas ajenas; aceptar y realizar críticas constructivas; colocarse en el lugar de otro de forma empática; respetar opiniones distintas a las propias con sensibilidad, etc.- necesarias para una buena convivencia y sana interacción.

\section{La metodología de aprendizaje servicio como potenciadora de competencias genéricas o transversales}

\section{El Aprendizaje Servicio como metodología universitaria}

El aprendizaje-servicio es un metodología pedagógica basada en la experiencia (Stanton, 1990), en la cual los estudiantes, docentes y miembros de una institución comunitaria o pública trabajan juntos para satisfacer las necesidades de esa comunidad; integrando y aplicando conocimientos académicos, alcanzando de esta forma, los objetivos instruccionales del curso. Para esto se usa la acción, la reflexión crítica y la investigación; y se forma a los estudiantes para convertirles en miembros contribuyentes de una sociedad más justa y democrática (Stephenson, Wechsler y Welch, 2003).

Entendemos, al igual que Rial (2010), el aprendizaje-servicio como una propuesta pedagógica que implica la realización de una acción solidaria protagonizada por los/las estudiantes, destinada a atender necesidades reales de una comunidad y planificada de forma integrada con los contenidos curriculares de aprendizaje. De este modo, el aprendizaje-servicio consiste en una propuesta educativa que combina procesos de aprendizaje y de servicio a la comunidad en un solo proyecto bien articulado, donde los participantes aprenden al trabajar en necesidades reales del entorno con la finalidad de mejorarlo (Puig y Palos, 2006). Además, el Aprendizaje Servicio sin dejar de ser un programa, es también una filosofía. Es decir, una manera de entender el crecimiento humano, una manera de explicar la creación de vínculos sociales y un camino para construir comunidades humanas más justas y con una mejor convivencia (Puig, 2007).

Los proyectos de aprendizaje-servicio permiten desarrollar los contenidos curriculares, es decir, aprendizajes académicos, pero además de una manera más clara y sencilla de lo que ocurre con otras metodologías, facilita la práctica de la planificación, el esfuerzo, la responsabilidad, el compromiso solidario... un sinfín de valores que de otra forma resulta complicado poder abordar desde una asignatura; a su vez, activa el ejercicio de todas las competencias del currículum, con énfasis en la iniciativa y autonomía personal y la competencia social y ciudadana. El aprendizaje aporta calidad al servicio y el servicio aporta significado y aplicación al aprendizaje (Tapia, 2008). El ApS tal y como indica Furco (2004) está orientado explícita y planificadamente a ofrecer un servicio solidario eficaz y a mejorar el aprendizaje de los estudiantes. Además, esta metodología logra aumentar significativamente los niveles de retención y aplicación de los contenidos de los cursos que la utilizan versus los que no lo hacen (Astin, Alexander et al., 2000).

Diversos estudios (Billig, Jesse y Root, 2006; Cabrera, 2002; Tapia, 2002) han demostrado que el aprendizaje-servicio (ApS) además de facilitar la adquisición de contenidos teóricos origina y promueve una serie de valores, como la responsabilidad y la solidaridad, necesarios en nuestra sociedad actual. 
Así mismo, gracias a esta metodología podemos trabajar eficazmente las competencias transversales o genéricas propias de todas las profesiones -aspectos genéricos de conocimientos, habilidades, destrezas y capacidades que debe tener cualquier titulado antes de incorporarse al mercado laboral- en las que los alumnos deben ser formados. Dichas competencias fueron definidas por la Agencia Nacional de Evaluación de la Calidad (ANECA) a partir del Proyecto Tuning -competencias instrumentales, competencias personales, competencias sistémicas y otras-.

\section{El proyecto Recreos cooperativos e inclusivos}

Comencemos contextualizando el proyecto. Este proyecto denominado "Recreos Cooperativos e Inclusivos" se inició como una experiencia piloto que llevamos a cabo el curso 2011-2012, desarrollada entre el CEIP Pedro J. Rubio de Huesca y la Facultad de Ciencias Humanas y de la Educación de Huesca.

En esta experiencia y tras la evaluación de la misma pudimos constatar como los estudiantes de Magisterio desarrollaron algunas competencias propias del título y a la vez brindaron un servicio a la comunidad educativa del entorno próximo a través de metodologías de cooperación. Concretamente, mejorando las interacciones que se establecían en los recreos entre los niños de primaria y disminuyendo la exclusión social que algunos niños sufrían en estos ratos de ocio.

Los resultados fueron tan exitosos el primer año que el curso 2012-2013 se sumó al proyecto otro colegio, el CEIP Pirineos-Pyrénées. En el curso 2013-2014 se incorporaron el CEIP el Parque y el CEIP Pio XII de la ciudad de Huesca. Y en el curso actual participan todos los centros públicos de Huesca, salvo uno, y algunos centros de la zona rural próxima.

Este proyecto es una experiencia de metodología de Aprendizaje Servicio (ApS) dónde los estudiantes de Magisterio desarrollan competencias del Título del Grado de Magisterio y los colegios que participan reciben una ayuda para resolver las dinámicas marginadoras y segregacionistas que se estaban estableciendo en los recreos y generar más interacciones positivas de las habituales. Se trata de un proyecto interdisciplinar pues se desarrollan juegos cooperativos en el recreo relacionados con diversas áreas como Educación Física, Educación Artística, Didácticas Específicas, etc.

Va dirigido a los estudiantes de Magisterio del Grado de Educación Primaria que realicen las prácticas escolares en los colegios que han solicitado nuestra colaboración y a los niños de Primaria de estos centros educativos.

Los estudiantes de Magisterio reciben formación teórico/práctica sobre aprendizaje y juego cooperativo por parte de profesionales de la Facultad, también reciben unas horas de formación práctica aprendiendo juegos de carácter cooperativo en la Ludoteca y además los orientadores y trabajadores sociales de los colegios implicados sensibilizan a nuestros estudiantes de la importancia que tiene su colaboración para mejorar las interacción entre los niños de primaria en los recreos. Una vez recibida esta formación en los colegios, en la Facultad y en la Ludoteca de la ciudad, en la hora del patio, los estudiantes de Magisterio ponen en práctica los juegos en grupos cooperativos con niños de primaria.

Es un proyecto dónde colabora el E.O.E.P. Hoya-Monegros (dos orientadores y una trabajadora social) y la ludoteca municipal, además de seis colegios de educación infantil y primaria de Huesca.

Es preciso indicar que se trata de un proyecto de gran interés tanto para la Institución Universitaria como para la Comunidad, por el apoyo brindado, así como para nuestros estudiantes que tienen la oportunidad de estar en contacto con niños de primaria en un 
contexto escolar lúdico. Por lo tanto, es un proyecto que encierra un enorme potencial en un doble sentido: por un lado, los alumnos aprenden de manera significativa, viendo utilidad y aplicabilidad a sus aprendizajes en situaciones reales y al mismo tiempo tocamos el tema de la responsabilidad social -compartir material para facilitar el trabajo de otros compañeros, el profesor como transformador social-. Así mismo, se da respuesta a una necesidad colectiva, dinamizar los recreos para mejorar la socialización, y crear nuevos lazos y relaciones positivas entre los alumnos de primaria de los colegios implicados en este proyecto.

\section{¿Por qué lo planteamos?}

Este proyecto surge ante la propuesta del orientador y la trabajadora social del Equipo de Orientación Educativa y Psicopedagógica de la Hoya de Huesca. Pues parece ser que era habitual en algunos colegios de nuestro entorno que la hora del "recreo", paradójicamente, era para algunos niños y niñas un momento de soledad en algunas ocasiones y de tensión en otras.

En estos niños se observa que intentan quedarse en el aula con cualquier excusa y si se les invitaba a bajar al patio solían estar solos, observando cómo jugaban los demás o juntándose con algún otro niño o niña de sus mismas características. Se puede decir que eran uniones "por exclusión" en lugar de "por elección o afinidad".

También se daba una casuística diferente consistente en niños con escasa 'competencia social' que, más que aislados, acababan rechazados porque su forma de relacionarse con los compañeros era inapropiada y basada generalmente en un repertorio de conductas bruscas, de saltarse las normas de los juegos y de recurrir a la fuerza física.

En ambos casos, bien sea por timidez o por lo contrario, la situación tendía a cronificarse y no era raro que permaneciera con ligeras variaciones a lo largo de toda la etapa de primaria. $\mathrm{Y}$ ello a pesar de las frecuentes intervenciones de tutores y tutoras para reconducir la situación mediante entrevistas tutoriales y mediaciones para la resolución de conflictos. Intervenciones que en bastantes ocasiones sí alcanzaban el objetivo deseado.

Otro factor a considerar, de importancia cada vez más acusada, era la preponderancia que en las actividades del patio tenía el fútbol. Se había adueñado de tal manera de los recreos que "barría" las pistas de baloncesto y de todo tipo de juegos, y en los colegios grandes se llegaban a habilitar tres y hasta cuatro zonas distintas para que los distintos cursos pudieran jugar sus partidos y sus "ligas".

En los colegios pequeños de la zona rural, al haber escaso número de alumnos, pasaba a ser la actividad prácticamente "única", y al chico que no le gustaba o no se le daba bien, quedaba condenado al ostracismo, cuando no le acompañara la etiqueta de "raro". En estas circunstancias, el presunto elemento de socialización se transforma en elemento de "exclusión".

Si bien este panorama no era generalizado, sí parece realista afirmar que en la mayoría de las aulas había algún alumno que experimentaba esta problemática. Y si nos paramos a pensar en los sentimientos que debe generar esta situación a lo largo de toda o buena parte de su escolaridad, y de cómo debe repercutir en su autoconcepto, convendremos en que vale la pena buscar formas educativas de abordar de manera sistemática este estado de cosas.

Nos dimos cuenta que los estudiantes de Magisterio que tenían que hacer sus prácticas en los colegios que participan en este proyecto, podían beneficiarse del mismo. 
Nos resultó tan interesante, que como ya se ha dicho anteriormente, que el año curso 20112012 se inició como Plan Piloto en el colegio Pedro J. Rubio de Huesca, el curso 2012-2013 se amplío a otro colegio de la ciudad el colegio Pirineos Pirineés. En el curso 2013-2014 se incorporaron dos colegios más el CEIP El Parque y el CEIP Pio XII al considerar necesario y beneficioso el implantar esta experiencia en sus centros. En la actualidad, el CEIP Alcoraz y San Vicente han decidido implementarlo también.

Partiendo de esta realidad y siendo conscientes de las ventajas de trabajar con la metodología de ApS -a través de experiencias de aprendizaje en el servicio, los estudiantes de magisterio aprenden a ser profesionales reflexivos, sensibles a las cuestiones basadas en la comunidad. Aprenden a reflexionar de forma crítica acerca de sus propios pensamientos, aprendizajes y prácticas mientras trabajan con niños en contextos concretos (Buchanan, Baldwin, y Rudisill; 2008: 30)-surge la idea de preparar este proyecto.

Los objetivos que se persiguen en este proyecto en relación a la formación de los estudiantes de magisterio se concretan en:

-Formar maestros competentes para trabajar en escuelas inclusivas.

-Potenciar actitudes más inclusivas entre los futuros maestros.

-Contribuir a la formación integral de los estudiantes, académica y personal, potenciando el aprendizaje en valores, en derechos humanos, en acceso a la ciudadanía comprometida, es decir en responsabilidad social.

- Conectar teoría y práctica, acercando al alumnado a la realidad e implicándole en las necesidades de la misma.

- Incrementar la motivación e implicación del alumnado universitario.

- Potenciar el uso de metodologías cooperativas.

- Desarrollar estrategias y competencias que de otra forma es muy difícil adquirir por parte del alumnado (competencia comunicativa, de liderazgo, de relaciones interpersonales, emocional, social, ciudadana, etc.).

- Mejorar la integración y procesos de socialización en los centros educativos de infantil y primaria, potenciando los valores de convivencia, respeto, igualdad de sexos, no discriminación por razón de raza, ni condición física o psicológica, etc.

Y los objetivos que se persiguen con los alumnos de primaria son:

- Facilitar la inclusión de todos los estudiantes, especialmente orientado a aquellos en riesgo de exclusión.

- Potenciar la socialización facilitando la interacción de todos los niños a través del juego.

- Presentar juegos diversos que respondan los intereses y gustos de todos los niños, ampliando su repertorio.

\section{Participantes}

Durante el curso 2014/2015 han participado en el proyecto 38 alumnos de $3^{\circ}$ y 18 alumnos de $4^{\circ}$ del Grado de Magisterio de Educación Primaria. Los alumnos de cuarto están durante 10 semanas en el centro mientras que los de tercero permanecen 7 semanas. 
Los colegios que participan en el proyecto este curso son: CEIP Pedro J. Rubio, CEIP Pirineos Pyrénées, CEIP El Parque, CEIP Pio XII, CEIP Alcoraz, CEIP San Vicente todos ellos de Huesca y el CEIP Pedro I de Barbastro. Además, están implicados los miembros del Equipo de Orientación Educativa de Infantil y Primaria (E.O.E.I.P.) de Huesca, de cada uno de los colegios en los que se desarrolla el proyecto, la Ludoteca municipal de dicha ciudad y un profesor de la Facultad de Ciencias Humanas y de la Educación encargado de la formación previa de los alumnos.

Los destinatarios del servicio son los alumnos de primaria que determina cada uno de los colegios en función de las necesidades detectadas. Cada centro decide qué cursos son los que participan.

Remitiéndonos a los datos del curso 2013/12014, último curso del que ya tenemos elaborada la memoria, aunque participaban un número menor de colegios, los participantes son los siguientes:

- Colegio CEIP Pirineos: del curso $5^{\circ}$ y $6^{\circ}$ de Primaria el número de alumnos han sido 82 y 42 respectivamente.

$\mathrm{Y}$ estudiantes de magisterio participando en el proyecto 11 alumnos.

- Colegio CEIP PIO XII: $3^{\circ}$ y $4^{\circ}$ de Primaria, 64 alumnos.

$Y$ estudiantes de Magisterio participando en el proyecto 5 alumnos.

- $\quad Y$ en $1^{\circ}$ y $2^{\circ}$ de Primaria, 68 alumnos.

Y estudiantes de Magisterio participando en el proyecto 6 alumnos.

- Colegio CEIP Pedro J Rubio: $1^{\circ}$ y $2^{\circ}$ de Primaria: 133 alumnos.

Y estudiantes de Magisterio participando en el proyecto: 6 alumnos.

- Colegio CEIP El Parque: $5^{\circ}$ y $6^{\circ}$ de Primaria: 81 alumnos

$Y$ estudiantes de magisterio: 5 alumnos.

- $\quad 3^{\circ}$ y $4^{\circ}$ de Primaria: 68 alumnos.

Y 7 alumnos de magisterio.

El total alumnos de Educación Primaria que han participado en el proyecto, tal y como se puede observar, asciende a 538. De éstos entre el 2 y $5 \%$, dependiendo del contexto porque cada centro educativo es distinto, presentan dificultad de interacción social en el recreo. Las dificultades que principalmente se han detectado son: problemas de comunicación, aislamiento, baja autoestima, timidez, agresividad verbal y en menor medida física, ansiedad y absentismo escolar en algunas etnias, especialmente en la etnia gitana.

Además, en este proyecto participan un total de 40 alumnos de Magisterio.

\section{Diseño y planificación del proyecto: Recreos cooperativos e inclusivos}

Para llevar a cabo estos objetivos hemos diseñamos un proyecto, denominado Recreos Cooperativos e Inclusivos que constituye para nuestros estudiantes, como hemos comentado anteriormente, una experiencia de ApS. Los alumnos de la Facultad de Ciencias Humanas y de la Educación -futuros docentes- se ponen al servicio de los CEIP de Huesca, desarrollando juegos cooperativos en los recreos, con el fin de potenciar las relaciones sociales positivas entre los alumnos de primaria en situaciones de ocio. 
Teniendo en cuenta lo fundamental que es para el proyecto la programación del mismo, sistematizamos el proceso en diversas fases que se presentan a continuación y que nos han permitido operativizarlo. Vamos a presentar dichas fases en orden cronológico:

Fase1. Detección de necesidades. En junio de 2011 un grupo de profesoras junto con el equipo directivo de la Facultad, nos reunimos con el Equipo de Orientación Educativa y Psicopedagógica de Hoya de Huesca, para que nos expusieran los problemas de interacción social que presenciaban en los recreos de algunos centros educativos de Huesca.

Necesidad a la que responde el proyecto: desarrollar las competencias del título de Magisterio a través de metodologías más innovadoras y en contextos de prácticas reales y también mejorar las relaciones sociales que es establecen en muchos colegios de primaria en los recreos.

Fase 2. Contacto con los centros receptores del servicio. Tras delimitar y acordar las necesidades de los centros de Educación Infantil y Primaria del entorno próximo, diseñamos un calendario de acciones para el curso 2014-2015 para trabajar en los colegios.

Fase 3. Preparación. En esta fase se definieron los recursos necesarios para llevar a cabo el proyecto. Como primeras acciones que se han llevado a cabo nos encontramos con:

-Curso teórico de cuatro horas impartido por profesor de la Facultad sobre el juego cooperativo para nuestros estudiantes de Magisterio. Con el objetivo de capacitar a los alumnos en este tipo de metodologías.

-Formación de otras cuatro horas en la ludoteca de la ciudad, conociendo posibles juegos para trabajar en los recreos, basados en una metodología cooperativa.

-Formación de dos horas del orientador del centro sobre la finalidad de los recreos cooperativos.

-Sesión inicial entre la profesora coordinadora, el orientador y los alumnos en prácticas para presentarles las características de cada grupo de alumnos, los juegos a desarrollar en esa primera sesión, la explicación de las rutinas de funcionamiento y el uso de los espacios.

Fase 4. Sesiones de trabajo práctico con los alumnos de la Universidad. Se llevan a cabo en las prácticas escolares de nuestros estudiantes universitarios en los colegios que participan en esta experiencia. En el proyecto se realiza una sesión semanal de juegos cooperativos en el recreo, durante cinco semanas de prácticas. Los grupos de alumnos de Educación Primaria que participan en estas sesiones son seleccionados por el coordinador del colegio, siendo aquellos grupos donde mayores problemas de interacción social e inclusión existen.

Estas sesiones las diseñan los estudiantes de magisterios respondiendo a cada coyuntura, orientados por el coordinador y a partir de la formación inicial recibida en la ludoteca y en la facultad. Así mismo, son los encargados de dinamizar y evaluar estas sesiones de juego.

A continuación presentamos a modo de ejemplo, algunas de las actividades realizadas en los centros: 


\begin{tabular}{l|l|}
\hline Colegio Pedro J. Rubio, para 1 ciclo Primaria & Colegio Pirineos Pyrinees, para 6 Primaria \\
\hline - Juegos Cooperativos con Paracaídas & - El ovillo, la Tela de araña. \\
\hline $\begin{array}{l}\text { - Juego de los paquetes, de los trenecillosy de la } \\
\text { cadeneta. }\end{array}$ & - La anilla, Jirafas y elefantes. \\
\hline - Chocolate inglés, y arrancar cebollas. & El tesoro, Zorros, gallinas y culebras. \\
\hline - Juego del Gavilán, del Stop y del Simón dice & El Pañuelo, balón sentado, balón prisionero. \\
\hline - Pelota sentada y las estatuas. & - Paracaídas, polis y cacos. \\
\hline
\end{tabular}

Fase 5. Evaluación del proyecto.

Respecto a la evaluación contemplamos, por un lado, la satisfacción del alumnado de la Universidad en la participación de este tipo de proyectos, para ello hemos elaborado un cuestionario de satisfacción que cumplimentarán los alumnos al finalizar sus propuestas. Por otro lado, tendremos en cuenta diversos indicadores que pueden apuntar cómo ha funcionado el proyecto: participación, asistencia, implicación, resultados de aprendizaje, etc. Así mismo se han vinculado varios TFG que han evaluado el impacto de dicho proyecto.

Fase 6. Difusión del proyecto. Nuestro compromiso es difundir este proyecto en toda la comunidad escolar para darle la máxima proyección posible y ampliarlo al mayor número de centros escolares y así incrementar el número de participantes. La difusión se hace en los medios locales pero también en jornadas y congresos nacionales e internacionales.

\section{Evaluación de Proyecto}

La evaluación del proyecto se realiza en distintos momentos y con distintos instrumentos.

a) Evaluación de las actividades de cada recreo: Se realiza en las reuniones de coordinación que cada colegio tiene y que está formado por el profesor coordinador del proyecto, por la persona del Equipo de Orientación Educativa de Infantil y Primaria (E.O.E.I.P.) del Centro y por los alumnos de la Facultad que participan. Se realizan cada semana y se valora cómo han resultado los juegos que se han realizado y las modificaciones que tienen que llevar a cabo además de programar el siguiente recreo.

b) Encuesta a los alumnos de Educación Primaria que han participado, al final del periodo de recreos cooperativos.

c) Encuesta a los alumnos de la Facultad de Ciencias Humanas y de la Educación después de que han acabado su periodo de prácticas con recreos cooperativos en el colegio.

d) Valoración del colegio en su informe /memoria a final de curso donde se integran los resultados de las encuestas anteriores más la valoración de los profesores del ciclo al que pertenecían los alumnos.

e) Memoria de los Recreos cooperativos que realizan los alumnos de Magisterio y que se entrega en la Facultad a los coordinadores del proyecto.

f) Reunión de evaluación /programación de las personas responsables del proyecto en la Facultad y el Equipo de Orientación Educativa de Infantil y Primaria (E.O.E.I.P.) de Huesca que valoran los resultados y programan el curso siguiente. 


\section{Resultados}

La valoración general de todos los agentes implicados ha sido muy positiva por lo que para futuro deseamos poder continuar con la experiencia. Durante estos recreos se han dado cambios en las actividades, en las relaciones y se ha potenciado más interacciones que las habituales -cantidad y variedad-. Algunos de los datos que nos permiten realizar esta afirmación son los siguientes:

- Las tutoras y monitores dicen que cuentan con una experiencia de interacción exitosa.

- Hubo algunos alumnos absentistas que asistían más al colegio el día de los juegos.

- Sigue dándose separación entre chicos y chicas en los juegos. Pero los alumnos acudían expectantes a la actividad y decían que les gustaba.

- Otro aspecto a valorar ha sido la introducción de juegos nuevos en el patio del recreo, diversificando la oferta y superando en cierto modo, el monopolio del fútbol como juego estrella.

- Aunque los niños sin problemas de relación son los que mejor valoran la actividad, en el desarrollo de la misma se observa a los niños poco integrados también contentos.

En lo que respecta a la valoración que realizan los alumnos los alumnos de Primaria cabe indicar que:

- El 90\% valoran la relación interna del juego como muy buena, tan sólo hay un alumno que la valora como mala.

- $\quad$ El 100\% dice que se lo han pasado muy bien jugando entre todos.

- En un 70\% dicen que los juegos cooperativos han disminuido las peleas.

- Un 50\% afirma que se produce un cambio en las rutinas de todos los recreos.

- Un $40 \%$ se siente a gusto de poder ayudar a algún compañero y de que lo ayuden a él.

- Un 80\% dice que ha tratado con compañeros que no conocía.

- Un 70\% han descubierto una nueva forma de pasarlo bien.

Las mejoras obtenidas respecto a la situación de partida en los estudiantes de primaria de los colegios participantes se concretan en: los chicos participen en estos juegos cooperativos en los recreos favorece el que se creen nuevos lazos y relaciones entre los alumnos, se mejora la cohesión como grupo aula, se evita la competitividad y se juega sin la necesidad de buscar "ganadores ni perdederos" se aprende a jugar de manera cooperativa, se disminuyen las peleas en el los recreos y el aislamiento de algunos alumnos, se cambian las rutinas en los recreos, se disfruta ayudando a alumnos con necesidades educativas especiales, se disminuye el absentismo escolar, entre otras. Además de la mejora de las interacciones que se producen en los recreos de los colegios entre los niños de primaria se potencia los valores de convivencia, respeto, integración, igualdad de sexos, no discriminación por razón de raza ni condición física o psíquica, etc.

Por otra parte, las mejoras en nuestros estudiantes de Magisterio son evidentes, pues el participar en este proyecto les permite estar en contacto con la realidad del recreo de una escuela, conocer y poder observar las dinámicas que se ponen en marcha en dichos recreos, 
conocer y aprender a diseñar y a implementar juegos que potencian las relaciones cooperativas y no competitivas, y además con esta experiencia se desarrollan muchas competencias del título. Por ejemplo, los alumnos de la Facultad mejoraron sustancialmente sus conocimientos sobre el diseño y uso de los juegos cooperativos puesto que pudieron poner lo aprendido en práctica bajo la tutela de los Orientadores y los profesores de los colegios preparando con ellos las intervenciones y evaluando el resultado de las mismas.

\section{Conclusiones}

Este proyecto, que empezó como una experiencia piloto se ha extendido por toda Huesca $y$ en el futuro tiene la posibilidad de poder continuar y expandirse a otros colegios que están interesados en participar por estar sufriendo en los recreos la misma problemática. Creemos que será así, pues el proyecto está teniendo una valoración muy positiva en la comunidad escolar del entorno. Y consideramos este hecho como un indicador de éxito.

Parece ser que es habitual en cualquier colegio que la hora del "recreo", paradójicamente, es para algunos niños y niñas un momento de soledad en algunas ocasiones y de tensión en otras. Son niños que intentan quedarse en el aula con cualquier excusa y si se les invita a bajar al patio suelen estar solos, observando cómo juegan los demás o juntándose con algún otro niño o niña de sus mismas características -se puede decir que son uniones "por exclusión" en lugar de "por elección o afinidad"-. En algunos colegios ya se está trabajando el "día sin balón" puesto que parece que el dominio del balón es otro de los factores de discriminación en el recreo, sin embargo nuestro proyecto va más allá y ha tenido una gran aceptación.

La experiencia nos dice que los proyectos de ApS resultan muy positivos, son una buena herramienta de enseñanza-aprendizaje. A través de ella se aglutina el aprendizaje de diferentes competencias, como son el trabajo en equipo interdisciplinar, las habilidades en las relaciones personales, el compromiso ético o el razonamiento crítico (ANECA, 2005). Coincidimos con Marta Lazo y Gónzalez (2012) en que las prácticas de ApS en las que se dan cita diferentes métodos de aprendizaje activo, como el aprendizaje experiencial, el cooperativo o el constructivismo, se ajustan a los fundamentos pedagógicos demandados en el Espacio Europeo de Educación Superior (EEES), donde competencias genéricas o transversales constituyen una de las exigencias formativas.

Tras la experiencia realizada hemos detectado que gracias a esta metodología los alumnos pueden adquirir y desarrollar competencias, concretamente competencias transversales, que desde una metodología más tradicional difícilmente lo podrían hacer. Porque el aprendizaje-servicio permite aplicar lo estudiado en situaciones reales y motiva a desplegar la creatividad e iniciativa, promueve el compromiso personal y social -conducta prosocial-, incrementa la autoestima y la identidad, alimenta la empatía interpersonal y social, estimula las actitudes y habilidades para la comunicación, promueve la colaboración, etc. entre otras competencias.

Como conclusiones de este proyecto se puede decir que los alumnos de la Facultad mejoraron sus conocimientos y desarrollan competencias transversales.

Por su parte los alumnos de primaria gracias a la intervención que mejora las interacciones que se producen en los recreos, adquieren valores de convivencia, respeto, solidaridad, igualdad de sexos, etc. valores esenciales para la inclusión.

También hemos analizado algunos aspectos sobre los que se trabajará en próximos cursos para incidir en la mejora del proyecto, como: 
- Buscar más juegos que favorezcan las conductas prosociales, por lo que el próximo curso al seleccionar con la ludoteca los juegos buscaremos potenciar este aspecto.

- Algunos colegios decían que sus recreos son pequeños, por lo que para el próximo curso a la hora de seleccionar los juegos tendremos en cuenta sus espacios e infraestructuras.

- Los colegios solicitan que más alumnos de Magisterio para futuro colaboren en este programa, pues lo consideran muy útil.

Por todo lo anterior, entendemos que el aprendizaje-servicio constituye hoy en día una de las principales vías metodológicas para fomentar el aprendizaje global y significativo en los alumnos, desarrollando todas las competencias transversales, en definitiva, una metodología por y para la formación integral de los alumnos.

Además, hemos conseguido crear una red de trabajo y colaboración entre la universidad y los centros educativos que podemos utilizar para futuras colaboraciones y que es tan necesaria. Porque desde la Facultad firmamos nuestra responsabilidad con nuestro entorno, por ello nos parece esencial establecer compromisos y crear redes de colaboración entre Escuela y Universidad, que a nuestro parecer son fundamentales para el incremento de la calidad de los estudios que ofertamos, acercando la realidad a las aulas y haciendo que nuestros alumnos aprendan significativamente (Vázquez, Gayán, Liesa, y Arranz, 2013).

Por lo expuesto y desde nuestro punto de vista, el aprendizaje-servicio puede resultar notablemente estimulante y enriquecedor para el alumnado, así como también para la sociedad (no sólo para el entorno próximo), ya que en última instancia esta metodología contribuye a la formación integral de los estudiantes universitarios -una formación de carácter integral que atienda tanto a contenidos disciplinares, profesionales, competenciales y actitudinales (Ferran y Guinot, 2012)- que son, obviamente, parte de la ciudadanía. En definitiva, responde a muchos de los retos que se plantean en la sociedad actual y en el nuevo marco Europeo de Educación Superior, promoviendo una de las funciones básicas de la Universidad: la formación de ciudadanos críticos, activos y responsables con su entorno (Francisco y Moliner, 2010).

\section{Referencias}

Agencia Nacional de Evaluación de la Calidad y Acreditación (2005). Libro blanco para el título de grado en magisterio. Madrid: ANECA.

Astin, A. W. et al. (2000). How Service Learning Affects Students. UCLA: Higher Education Research Institute.

Billig, S.; Jesse, D. y Root, S. (2006). The impact of service-learning on high school students' civic engagement. Evaluation report prepared for the Carnegie Corporation of New York. Denver: RMC Research Corporation.

Buchanan, A. M., Baldwin, S. C. y Rudisill, M. E. (2002). Service learning as scholarship in teacher education. Educational Researcher, 31 (8), 30-36.

Cabrera, F. (2002). Hacia una nueva concepción de la ciudadanía en una sociedad multicultural. En Bartolomé (Coord.), Identidad y Ciudadanía: Un Reto a la Educación Intercultural (pp. 49-76). Madrid: Narcea.

Francisco, A. y Moliner, L. (2010). El Aprendizaje Servicio en la Universidad: una estrategia en la formación de ciudadanía crítica. REIFOP, 13 (4), pp. 69-77 
Ferran, A. y Guinot, C. (2012). Aprendizaje-servicio: propuesta metodológica para trabajar competencias. Portularia, XII, 460-479.

Furco, A. (2004). El impacto educativo del aprendizaje-servicio. Ponencia en el VII Seminario Internacional de aprendizaje y servicio solidario. Buenos Aires, 6-7 octubre de 2004 (Ministerio de Educación, Ciencia y Tecnología, República Argentina. Disponible n: (www.me.gov.ar/edusol) [Consulta: 25 de enero 2013]

Marta Lazo, C. y Gónzalez Aldea, P. (2012): El aprendizaje-servicio, una herramienta para el desarrollo profesional de la responsabilidad social del periodista. Estudios sobre el Mensaje Periodístico, 18, 577-585.

Puig, J.; Batlle, R.; Bosch, C. y Palos, J. (2007). Aprendizaje Servicio: educar para la ciudadanía. Barcelona: Octaedro. Disponible en: http://www.zerbikas.es (primer capítulo) [Consulta: 12 de junio 2014]

Pujolàs Maset, P. (2012). Aulas inclusivas y aprendizaje cooperativo. Educatio Siglo XXI, 30 (1), 89-112.

Rial, S. (2010). Criterios de calidad y rasgos característicos de las experiencias de aprendizajeservicio en la educación formal. Tzhoecoen. Perú: Universidad Señor de Sipan de Chiclayo.

Stanton, T. (1990). Service learning: Groping toward a definition. En J. C. Kendall et Al. (Coords.) Combining Service and Learning: A Resource Book for Community and Public Service (Vol. 1) (pp. 65-67). Raleigh, NC: National Society

for Internships and Experiential Education.

Stephenson, M., Wechsler, A. y Welch, M. (2002). Service Learning in the Curriculum: a faculty guide. Utah: Lowell Bennion Community Service Center at the University of Utah.

Tapia, M. N. (2002). Service-learning in Latin America. Buenos Aires: CLAYSS.

Tapia, M.N. (2008). Calidad académica y responsabilidad social: el aprendizaje servicio como puente entre dos culturas universitarias. En Martínez, M. (Coord). Aprendizaje servicio y responsabilidad social de las Universidades (pp. 27-56). Barcelona: Octaedro

Vázquez, S; Gayán , T. Liesa, M. y Arranz, P. (2013). ApS y atención a la diversidad: SAACPRENDER innovación, inclusión y formación. En Vigo, B. y Soriano, J. (Coords.), Educación Inclusiva y formación del profesorado (pp. 392-400). Zaragoza 